\title{
AI Techniques and Mathematical Modeling to Detect Coronavirus
}

\author{
Kashif Ahmed $^{1}$ (i) Niharika Gouda $^{2,3}$
}

Received: 15 August 2020/ Accepted: 28 October 2020/Published online: 16 November 2020

(C) The Institution of Engineers (India) 2020

\begin{abstract}
The epic coronavirus (COVID-19) flare-up, distinguished in late 2019, requires unique consideration in view of its future pandemics and conceivable worldwide dangers. In addition to clinical methodology and medicines, artificial intelligence (AI) guarantees another worldview for social welfare, a few distinctive AI methods that are based upon machine learning calculations are utilized for investigating information and dynamic procedures. This paper presents a survey on various mathematical model and AI-based devices to understand its growth which could assist in ruling out COVID-19 infection. Also, a new technique for the forecast of the disease is investigated. These methods if inculcated in practice can help in curtailing the further spread of the disease.
\end{abstract}

Keywords Artificial intelligence · COVID-19 .

Forecasting · Mathematical modeling · Pneumonia .

$\theta$-SEIHRD

\section{Introduction}

COVID-19 short for Coronavirus disease 2019 is a highly contagious disease originated in Wuhan province of China in December 2019. World Health Organization (WHO)

Kashif Ahmed

kashifahmedy2k10@gmail.com

Niharika Gouda

niharikagouda92@gmail.com

1 Department of Electrical and Electronics Engineering, CMR Institute of Technology, Bengaluru, India

2 Università degli Studi dell'Aquila, L'Aquila, Italy

3 Amrita Vishwa Vidyapeetham, Bengaluru, India conceded that virus can create respiratory ailment with fever and cough. Without a remedial vaccine or explicit antiviral medications, early identification tools and separation becomes essential against novel coronavirus. World Health Organization council announced COVID-19 as a pandemic because of quick individual-individual spread and affected patients have less immunity. The coronaviruses come in the group of Coronaviridae. The infections cause moderate virus Middle East respiratory syndrome (MERS) or Severe Acute Respiratory Syndrome to the individuals. COVID-19 is fervid infection brought about by SARS-CoV, which had begun in China and it was unfurled in numerous nations around the world [1]. The COVID-19 spread quickly in China and leaded to more than 7050 cases toward the finish of January [2]. The count of the initial month total cases of the COVID-19 has crossed the complete count of the SARS cases in 2003 [3]. As a result, the patients are outnumbering the medical staff. Accordingly, this makes medical organization and doctors over-burdened. This prompt delayed examination and isolation of affected individuals and low productive medication of patients.

\section{The Comparison Between COVID-19 and SARS- 2003}

In Fig. 1a [4] the count of cases of SARS in 2003 indicated in blue circle line and the COVID-19 in 2020 indicated in red circle line, where the broad blue line is the prediction of SARS 2003.

Figure $1 \mathrm{~b}$ [4] shows the rate of infection $\beta(t)$ of SARS indicated in blue circles and COVID-19 indicated in red circles.

Presently, there are two essential kinds of COVID-19 tests. 

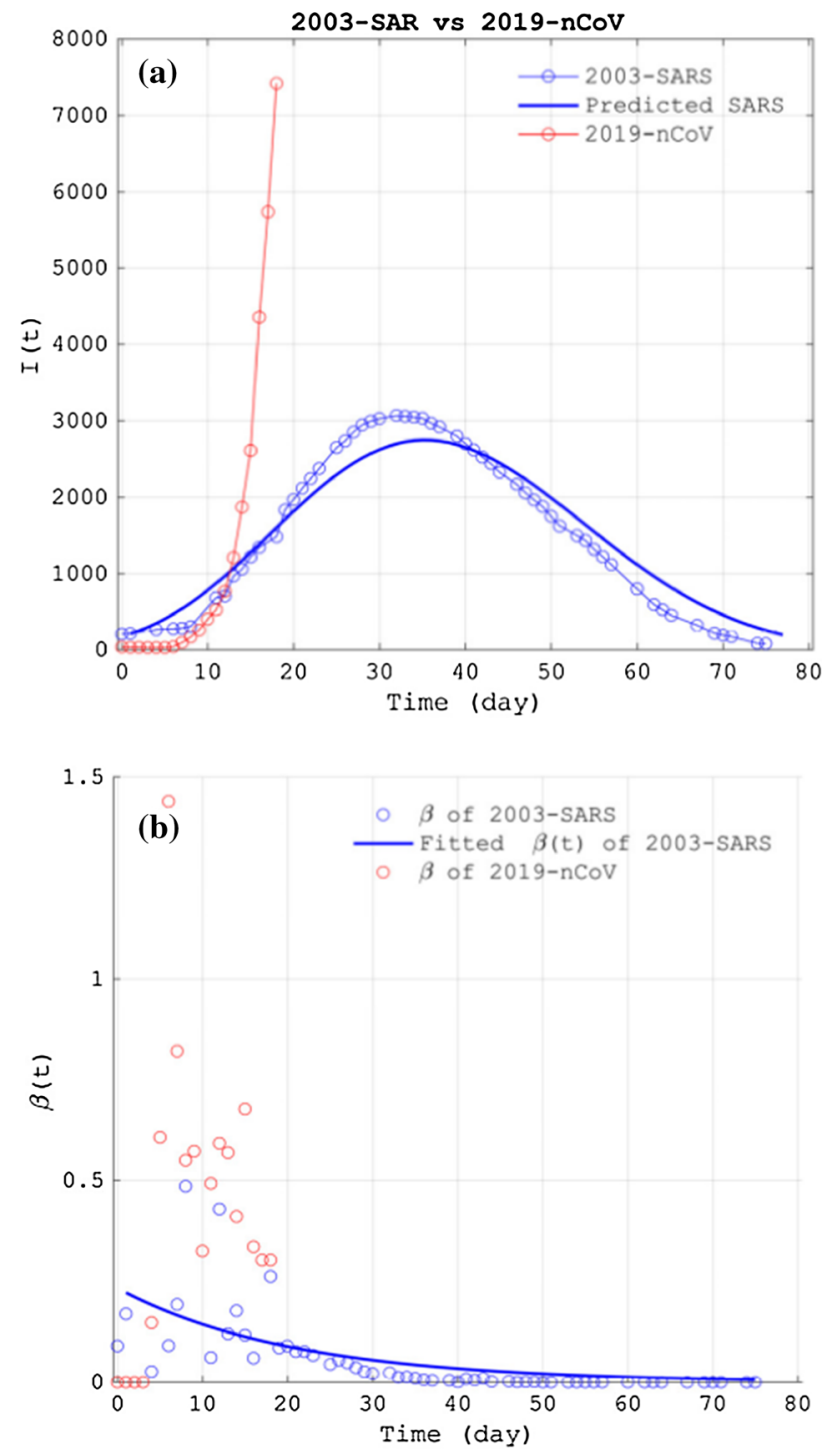

Fig. 1 a Count of cases of SARS-2003 vs. COVID-19 [4]. b Infection rates SARS-2003 vs. COVID-19 [4]

(1) The test screens a patient's blood tests for antibodies against the infection. However, antibodies are often not detectable until a few days after symptoms begin.

(2) The test searches for viral DNA in mouth or nasal swabs. While these can distinguish the infection prior, they take a few hours to perform.

\section{AI and Its Use in Coronavirus}

Pilot test checks of the infection is imperative in treatment and stop spread of the Virus. AI is useful in identifying of the contaminated cases with the assistance of clinical imaging advancements such as computed tomography (CT) and magnetic reverberation imaging (MRI) [5]. Figure 2
[5] shows comparison of AI-based and conventional treatment given to a person affected by Covid.

\section{Models and Techniques}

This segment presents the motivation and the point by point plan of the strategies. Figure 3 shows the model for coronavirus.

\section{AI Methods to Identify Coronavirus using Phone Detectors Method}

Medical team or individuals utilized this arrangement who own the smartphones and can be used anytime, anyplace. Accordingly, such model is required in the crisis circumstances [6].

The indications of the affirmed coronavirus patient ought to be figured it out. The common symptoms are shortness of breath, nausea, fever and fatigue. Indications of coronavirus are different from other indications of diseases like flu, cold, and fever. Thus, the system is attempting to find the degree of each indications on measurements of sensors. Also, many advance sensors are implanted in smart system including cameras, microphone, inertial sensors and temperature sensor etc. Then the sensor response is used for indications. For instance, temperature-fingerprint sensor is used to check the feverlevel of a human body. Images and videos taken from camera are used to figure out human fatigue. Camera and inertial sensors are used to check the neck posture and level of headache.

The calculations of Sensor measurement give the results of infection which is put in dataset as a single record used as an input for further prediction by Machine Learning algorithm. [7]. Along these lines, details of infected persons are gathered and utilized as source in AI model.

Few AI methods like decision tree, SVM, and neural networks are implemented. Many profound learning calculations are used for characterization needs such as convolution neural system $(\mathrm{CNN})$ and repetitive neural system (RNN) [8].

For identifying coronavirus, CT (scanned pictures) imaging of lungs is main strategy. A method is created to analyze the lesions of affected persons and investigating the proportion. Output of the method is the volume.

\section{Detection of Coronavirus Using $\mathrm{X}$-ray by Neural Networks}

AI methods and X-ray together can be used in tandem for the accurate detection of this illness and can likewise be helpful to solve the issue of absence of specific doctors in 
Fig. 2 Non-AI and AI methods to detect coronavirus [5]

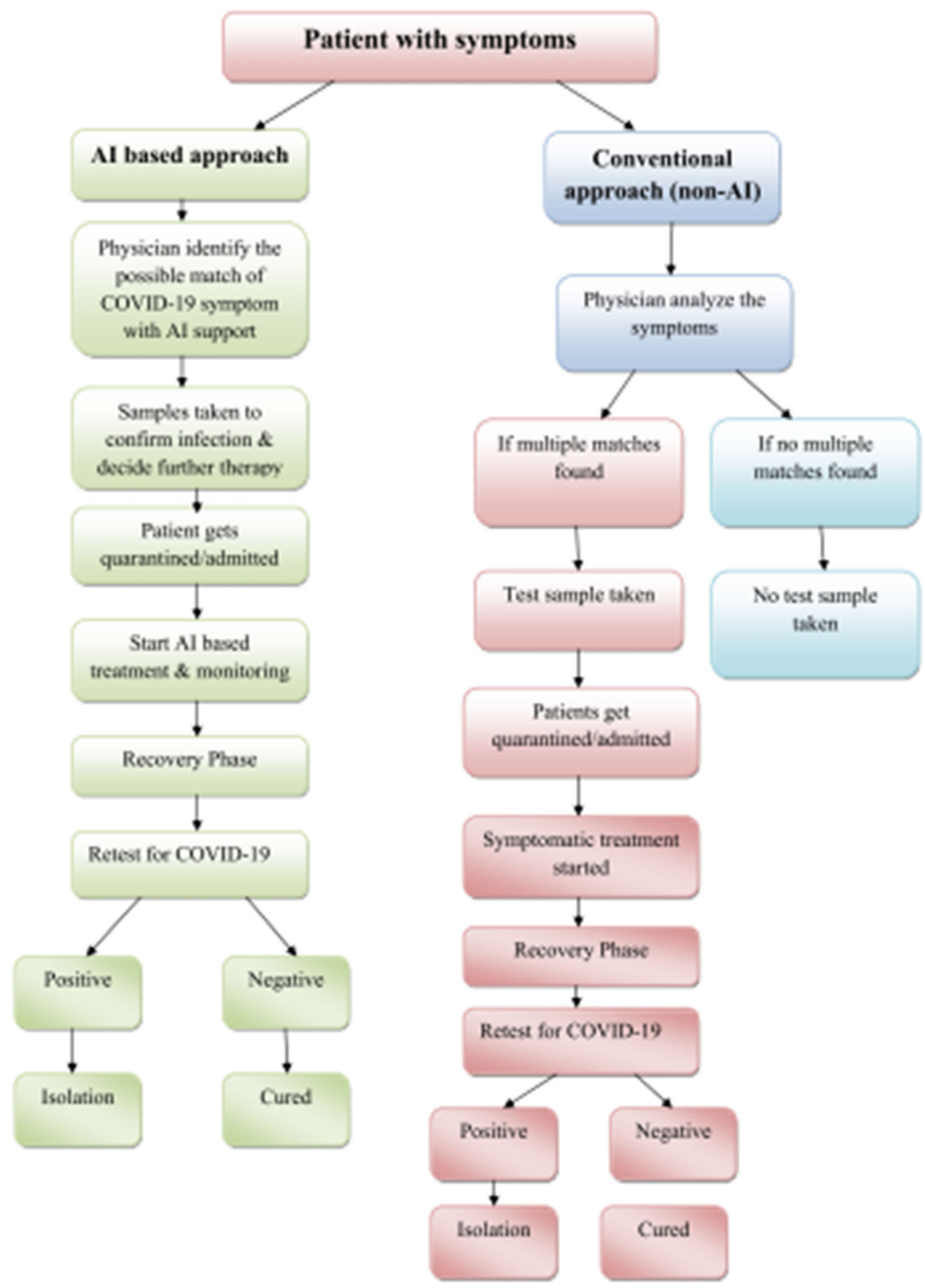

small towns. This approach is helpful in providing precise treatment of multi-class and binary classification. A total of 1125 images (125 COVID-19(+), 500 Pneumonia and 500 No-Findings) were used [9]. Modeling the real-time object recognition framework is done by a system called YOLO (You only look once), which is a classifier method as Darknet-19. This classifier contains 5 pooling layers and 19 convolutional layers. The layer layout is like:

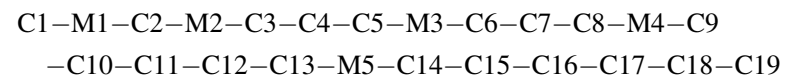

Two type of scheme are implemented to distinguish and analyze coronavirus by X-ray.

1. DarkNet-19 method is trained and is helpful to distinguish visuals to groups:

- COVID-19

- No-Findings

- Pneumonia 


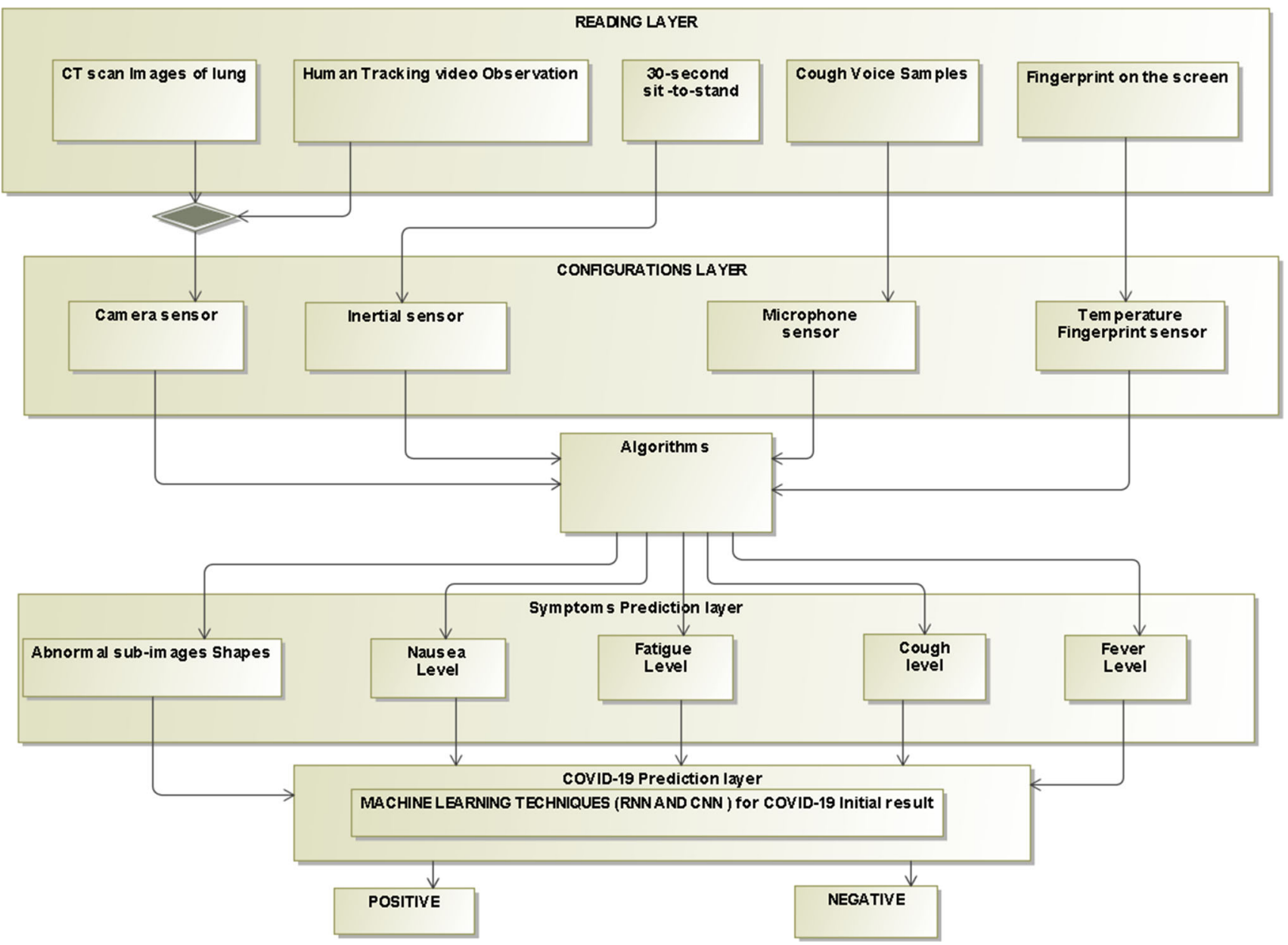

Fig. 3 Model for detection of coronavirus

2. Classification of two classes can be done after training the DarkNet-19 method:

- COVID-19 and

- No-Findings

The efficiency of this method is assessed utilizing the 5-overlay cross-approval methodology for these two-characterization issue. $80 \%$ of $\mathrm{X}$-beam pictures are utilized for training and $20 \%$ for verification. The tests are done multiple times.

Results of both type of grouping of method are assessed for every overlay, and the normal order efficiency is determined. Figure 4 [10] shows layers of DarkNet-19 architecture.

\section{Multi-class Classification and Binary-class Classification}

Figure 5a, b [10] represents the confusion matrix result for multi-class and binary-class classifications, respectively. From Tables 1 and 2 [10], multi-class framework was found to be able to perform classification with an accuracy of 87.02 percent and binary framework was found with a precision of 98.07 percent. This has been corroborated by experts in radiology and the same is ready to be rolled out to a larger database.

\section{Mathematical Formulation of Model}

The formulation is based on $\theta$-SEIHRD design [11]. Features of this design are:

- Susceptible (symbol S) The individual is not contracted from infection.

- Exposed (symbol E) The individual is in the development stage of being infected and has no indications. The individual could contaminate others, however, with a lower likelihood than individuals in the infectious category.

- Infectious (symbol I) Initial category of the stage. Individual may contaminate others and starts showing indications. Individuals in this category are controlled by hospital specialists or remains un-recognized. 


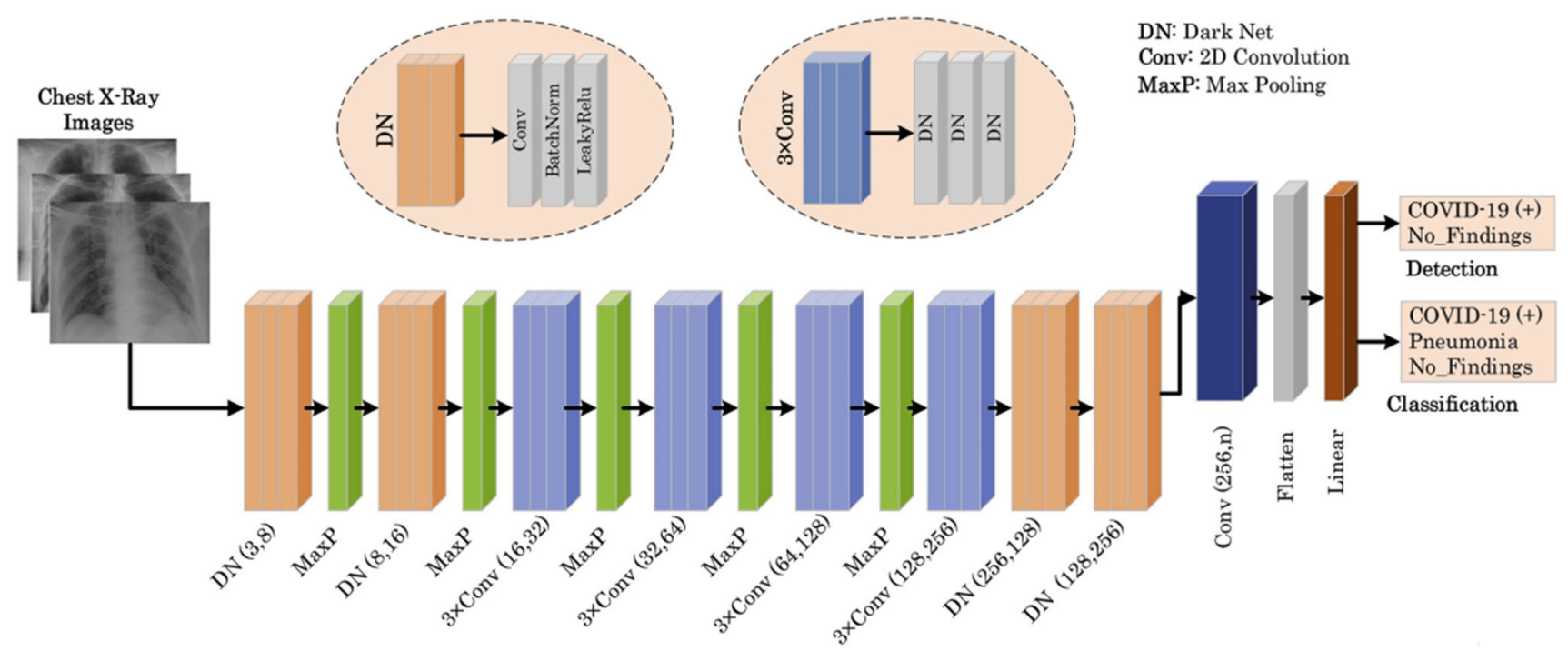

Fig. 4 DarkNet-19 architecture [10]

- Infectious but undetected (symbol $I_{u}$ ) Individual can at present contaminate others and have indications, however, are not distinguished.

- Hospitalized (symbol $H_{R}$ ) Individuals in emergency clinic can still contaminate others. Patients have possibility to recover.

- Hospitalized that will die (symbol $H_{D}$ ) Individual is in emergency care and can still spread the disease to others.

- Dead by COVID-19 (symbol D) Individual could not survive the infection.

- Recovered after being previously detected as infectious $\left(\right.$ symbol $R_{d}$ ) Individuals were recently distinguished as infectious, but at this point is not infectious and has built up an immune system to the infection. Patient still needs to be taken care of after recovering.

- Recovered after being previously infectious but undetected (symbol $R_{u}$ ) Individual was infectious but at present isn't infectious and has built up a characteristic resistance to the Virus.

- Isolation Contaminated individuals are disengaged from contact with others. Just sanitary experts are in contact with them. Isolated patients get a sufficient clinical medication that diminishes the casualties.

The dynamic infection spread inside a specific affected region is demonstrated by utilizing a mathematical categorical method. Individuals in a domain are placed in one of those categories: $S, E, I, I_{\mathrm{u}}, H_{\mathrm{R}}, H_{\mathrm{D}}, R \mathrm{~d}, R \mathrm{u}$ or $D$.

Every time people in region is spread equally. (It can be improved by separating a few regions into a lot of littler areas with comparable qualities.) Hence, the spatial dispersion of the pandemic in region is not included. We likewise expect that new births are vulnerable individuals
[12]. Under those suppositions, below are the system of ordinary differential equations:

$$
\begin{aligned}
\frac{\mathrm{d} S^{(i)}}{\mathrm{d} t}(t)= & -\frac{S^{(i)}(t)}{N^{(i)}}\left(m_{\mathrm{E}}^{(i)}(t) \beta_{\mathrm{E}}^{(i)} E^{(i)}(t)+m_{\mathrm{I}}^{(i)}(t)\right. \\
& \left.\beta_{\mathrm{I}}^{(i)} I^{(i)}(t)+m_{\mathrm{I}_{\mathrm{u}}}^{(i)}(t) \beta_{\mathrm{I}_{\mathrm{u}}}^{(i)}\left(\theta^{(i)}(t)\right) I_{\mathrm{u}}^{(i)}(t)\right) \\
& -\mu_{\mathrm{m}}^{(i)} S^{(i)}(t)+\mu_{\mathrm{n}}^{(i)}\left(S^{(i)}(t)+E^{(i)}(t)+I^{(i)}(t)\right. \\
& \left.+I_{\mathrm{u}}^{(i)}(t)+R_{\mathrm{d}}^{(i)}(t)+R_{\mathrm{u}}^{(i)}(t)\right)
\end{aligned}
$$

$\frac{\mathrm{d} E^{(i)}}{\mathrm{d} t}(t)=\frac{S^{(i)}(t)}{N^{(i)}}\left(m_{\mathrm{E}}^{(i)}(t) \beta_{\mathrm{E}}^{(i)} E^{(i)}(t)+m_{I}^{(i)}(t)\right.$

$$
\left.\beta_{\mathrm{I}}^{(i)} I^{(i)}(t)+m_{\mathrm{I}_{\mathrm{u}}}^{(i)}(t) \beta_{\mathrm{I}_{\mathrm{u}}}^{(i)}\left(\theta^{(i)}(t)\right) I_{\mathrm{u}}^{(i)}(t)\right)
$$$$
+\frac{S^{(i)}(t)}{N^{(i)}}\left(m_{\mathrm{H}_{\mathrm{R}}}^{(i)}(t) \beta_{\mathrm{H}_{\mathrm{R}}}^{(i)} H_{R}^{(i)}(t)+m_{\mathrm{H}_{\mathrm{D}}}^{(i)}(t) \beta_{\mathrm{H}_{\mathrm{D}}}^{(i)} H_{\mathrm{D}}^{(i)}(t)\right)
$$$$
-\mu_{\mathrm{m}}^{(i)} E^{(i)}(t)-\gamma_{\mathrm{E}} E^{(i)}(t)+\tau_{1}^{(i)}(t)-\tau_{2}^{(i)}(t)
$$

$$
\frac{\mathrm{d} I^{(i)}}{\mathrm{d} t}(t)=\gamma_{\mathrm{E}} E^{(i)}(t)-\left(\mu_{\mathrm{m}}^{(i)}+\gamma_{\mathrm{I}}^{(i)}(t)\right) I^{(i)}(t)
$$

$\frac{\mathrm{d} I_{\mathrm{u}}^{(i)}}{\mathrm{d} t}(t)=\left(1-\theta^{(i)}(t)\right) \gamma_{\mathrm{I}}^{(i)}(t) I^{(i)}(t)-\left(\mu_{\mathrm{m}}^{(i)}+\gamma_{\mathrm{I}_{\mathrm{u}}}^{(i)}(t)\right) I_{\mathrm{u}}^{(i)}(t)$

$$
\begin{aligned}
\frac{\mathrm{d} H_{R}^{(i)}}{\mathrm{d} t}(t)= & \theta^{(i)}(t)\left(1-\frac{\omega^{(i)}(t)}{\theta^{(i)}(t)}\right) \gamma_{\mathrm{I}}^{(i)}(t) I^{(i)}(t) \\
& \left.-\gamma_{\mathrm{H}_{\mathrm{R}}}^{(i)}(t)\right) H_{\mathrm{R}}^{(i)}(t)
\end{aligned}
$$

$\frac{\mathrm{d} H_{\mathrm{D}}}{\mathrm{d} t}(t)=\omega(t) \gamma_{\mathrm{I}}(t) I(t)-\gamma_{\mathrm{H}_{\mathrm{D}}}(t) H_{\mathrm{D}}(t)$

$\frac{\mathrm{d} R_{\mathrm{d}}}{\mathrm{d} t}(t)=\gamma_{\mathrm{H}_{\mathrm{R}}}(t) H_{\mathrm{R}}(t)$ 
(a)

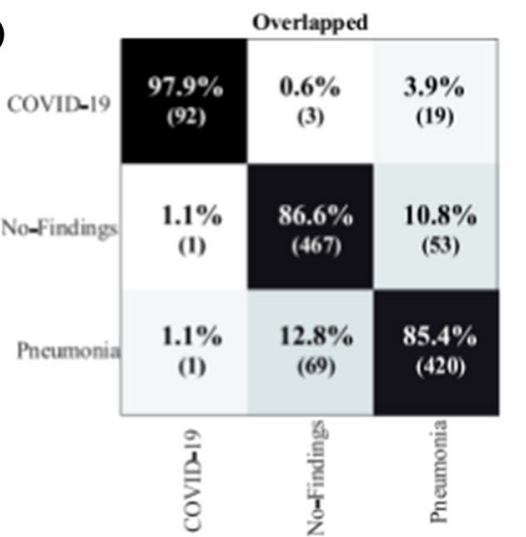

(a)

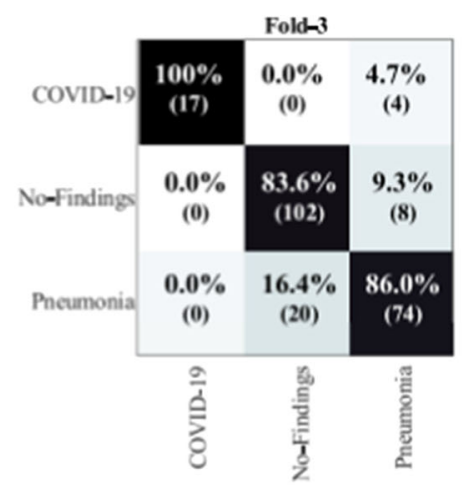

(d)

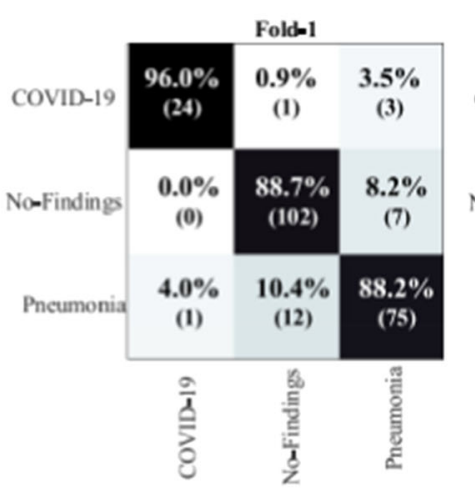

(b)

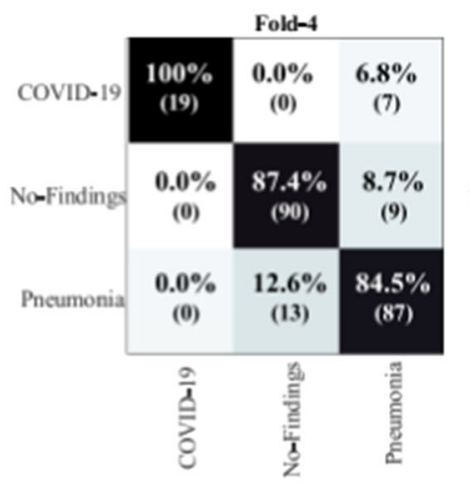

(e)
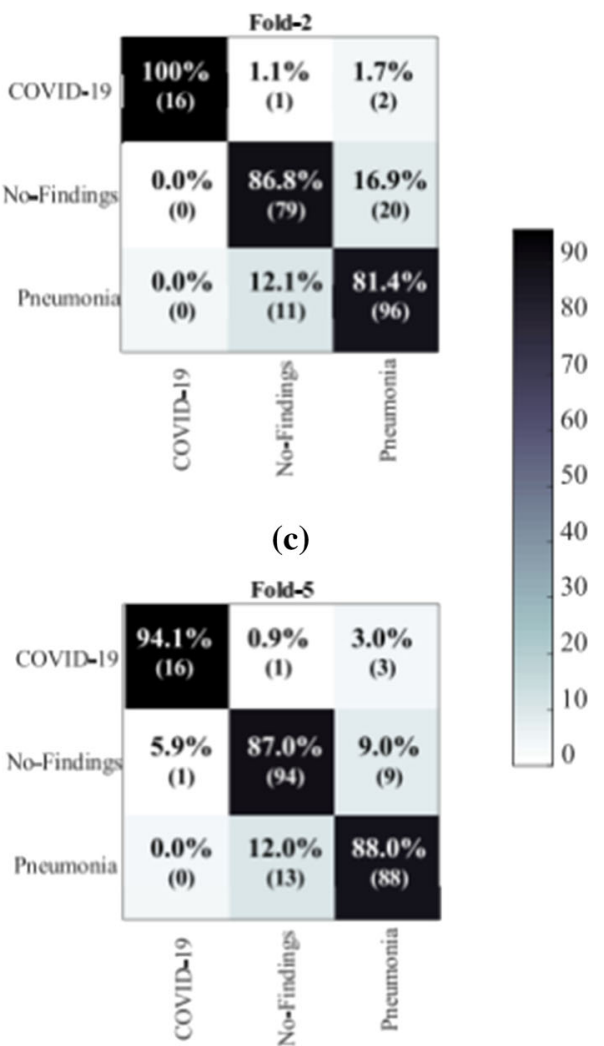

(f)

(b)

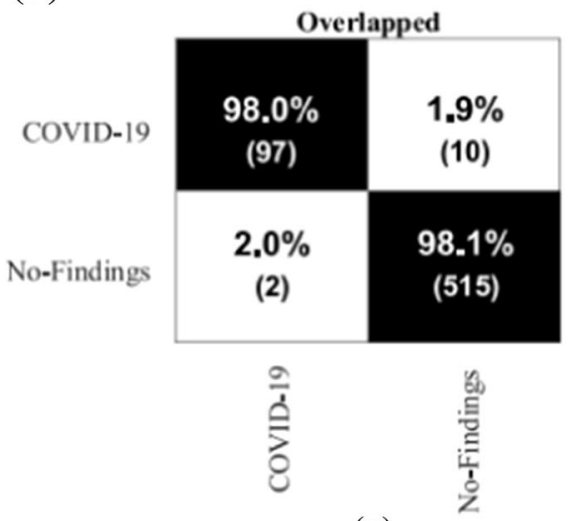

(a)

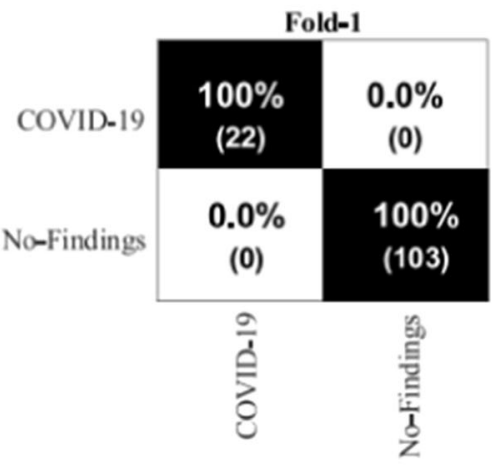

(b)

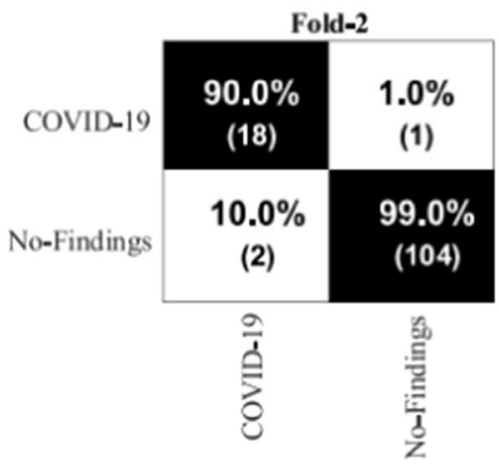

(c)

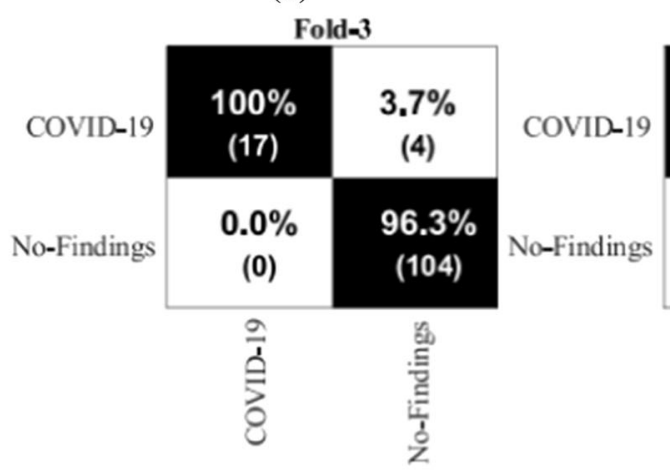

(d)

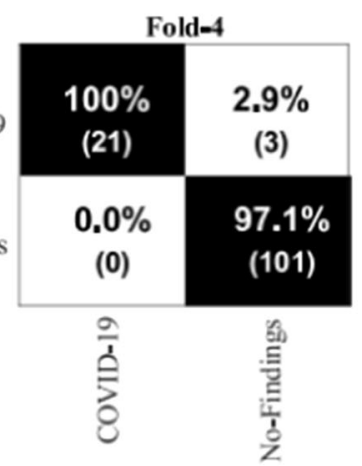

(e)

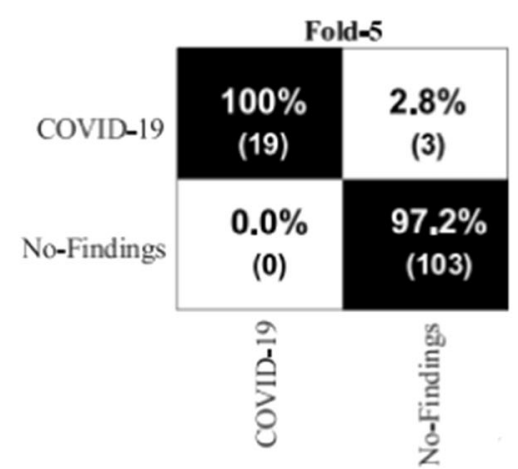

(f) 
Fig. 5 a Confusion matrix result of multi-class classification [10].

b Confusion matrix result of binary-class classification [10]
- $m_{E}^{(i)}, m_{I}^{(i)}, m_{I_{u}}^{(i)}, m_{H_{R}}^{(i)}, m_{H_{D}}^{(i)}$ : Performance of every category management.

- $\tau_{1}^{(i)}(t)$ : Affected individual visit area.

$\tau_{2}^{(i)}(t)$. Affected individual move from area.

Table 1 Performance of every overlay of multi-class classification [10]

\begin{tabular}{|c|c|c|c|c|c|}
\hline \multirow[t]{2}{*}{ Folds } & \multicolumn{5}{|c|}{ Performance metrics $(\%)$} \\
\hline & Sensitivity & Specificity & Precision & $F 1$-score & Accuracy \\
\hline Fold-1 & 88.17 & 93.66 & 90.97 & 89.44 & 89.33 \\
\hline Fold-2 & 84.57 & 90.61 & 89.38 & 86.63 & 84.89 \\
\hline Fold-3 & 84.13 & 91.14 & 89.88 & 86.54 & 85.78 \\
\hline Fold-4 & 83.66 & 92.29 & 90.61 & 86.42 & 87.11 \\
\hline Fold-5 & 85.83 & 92.75 & 89.71 & 87.57 & 88.00 \\
\hline Average & 85.35 & 92.18 & 89.96 & 87.37 & 87.02 \\
\hline
\end{tabular}

Table 2 Performance of every overlay of binary-class classification [10]

\begin{tabular}{|c|c|c|c|c|c|}
\hline \multirow[t]{2}{*}{ Folds } & \multicolumn{5}{|c|}{ Performance metrics (\%) } \\
\hline & Sensitivity & Specificity & Precision & $F 1$-score & Accuracy \\
\hline Fold-1 & 100 & 100 & 100 & 100 & 100 \\
\hline Fold-2 & 96.42 & 96.42 & 94.52 & 95.52 & 97.60 \\
\hline Fold-3 & 90.47 & 90.47 & 98.14 & 93.79 & 96.80 \\
\hline Fold-4 & 93.75 & 93.75 & 98.57 & 95.93 & 97.60 \\
\hline Fold-5 & 93.18 & 93.18 & 98.58 & 95.62 & 97.60 \\
\hline \multicolumn{6}{|l|}{ Overlapped } \\
\hline COVID-19 & 90.65 & 99.61 & 97.97 & 94.17 & 98.07 \\
\hline No findings & 99.61 & 90.65 & 98.09 & 98.84 & 98.07 \\
\hline Average & 95.13 & 95.3 & 98.03 & 96.51 & 98.08 \\
\hline
\end{tabular}

$\frac{\mathrm{d} R_{\mathrm{u}}}{\mathrm{d} t}(t)=\gamma_{\mathrm{I}_{\mathrm{u}}}(t) I_{\mathrm{u}}(t)$

$\frac{\mathrm{d} D}{\mathrm{~d} t}(t)=\gamma_{\mathrm{H}_{\mathrm{D}}}(t) H_{\mathrm{D}}(t)$

where

- $i$ : count of considered nations/domains.

- $\mu_{n}^{(i)}$ : rate of birth.

- $\mu_{m}^{(i)}$ : rate of death.

- $\omega^{(i)}(t)$ : ratio of count of deaths and affected individual.

- $\theta^{(i)}(t)$ : ratio of recognized affected individual and registered by experts.

- $\beta_{\mathrm{E}}^{(i)}, \beta_{\mathrm{I}}^{(i)}, \beta_{\mathrm{I}_{\mathrm{u}}}^{(i)}, \beta_{\mathrm{H}_{\mathrm{R}}}^{(i)}, \beta_{\mathrm{H}_{\mathrm{D}}}^{(i)}$ : rate of individual sickness interaction in their category.

- $\gamma_{E}$ : rate of growth from category $E$ to $I$.

- $\gamma_{\mathrm{I}}^{(i)}(t)$ : rate of growth from category $I$ to $I_{\mathrm{u}}, H_{\mathrm{R}}$ or $H_{\mathrm{D}}$.

- $\gamma_{\mathrm{I}_{\mathrm{u}}}^{(i)}, \gamma_{\mathrm{H}_{\mathrm{R}}}^{(i)}, \gamma_{\mathrm{H}_{\mathrm{D}}}^{(i)}$ : rate of growth from category $I_{\mathrm{u}}, H_{\mathrm{R}}$ or $H_{\mathrm{D}}$ to $R_{\mathrm{u}}, R_{\mathrm{d}}$ and $D$.
We comment that, with this simplification, the last three conditions of the framework are not combined with different conditions. In this manner, computation occur between initial six conditions and result of ending three conditions.

$R_{\mathrm{d}}(t)=R_{\mathrm{d}}\left(t_{0}\right)+\int_{t_{0}}^{t} \gamma_{\mathrm{H}_{\mathrm{R}}}(s) H_{\mathrm{R}}(s) \mathrm{d} s$

$R_{\mathrm{u}}(t)=R_{\mathrm{u}}\left(t_{0}\right)+\int_{t_{0}}^{t} \gamma_{\mathrm{I}_{\mathrm{u}}}(s) I_{\mathrm{u}}(s) \mathrm{d} s$

For the numerical simulations, the initial conditions are computed by 4 th form Runge-Kutta technique.

\section{Results of Design}

The outcome of the method is shown with the above procedure and system of equations. 
Fig. 6 Pictorial analysis of mathematical model of COVID19 [11]

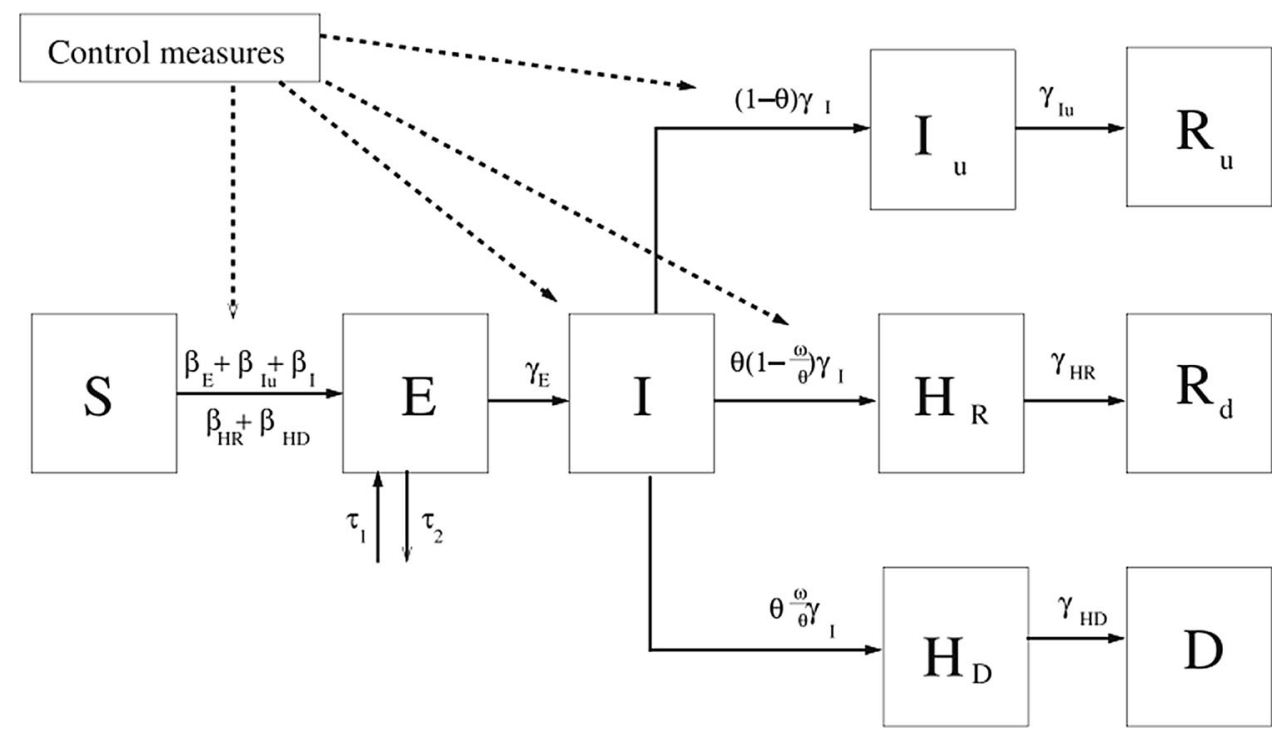

Table 3 Future predictions of Coronavirus cases of 10 days in India

\begin{tabular}{lllll}
\hline & ds & yhat & yhat_lower & yhat_upper \\
\hline 1 & $2020-07-25$ & $1.276593 \mathrm{e}+06$ & $1.221894 \mathrm{e}+06$ & $1.327947 \mathrm{e}+06$ \\
2 & $2020-07-26$ & $1.303652 \mathrm{e}+06$ & $1.251894 \mathrm{e}+06$ & $1.359976 \mathrm{e}+06$ \\
3 & $2020-07-27$ & $1.330136 \mathrm{e}+06$ & $1.281300 \mathrm{e}+06$ & $1.381636 \mathrm{e}+06$ \\
4 & $2020-07-28$ & $1.350382 \mathrm{e}+06$ & $1.295558 \mathrm{e}+06$ & $1.401771 \mathrm{e}+06$ \\
5 & $2020-07-29$ & $1.377298 \mathrm{e}+06$ & $1.325360 \mathrm{e}+06$ & $1.432255 \mathrm{e}+06$ \\
6 & $2020-07-30$ & $1.404414 \mathrm{e}+06$ & $1.352719 \mathrm{e}+06$ & $1.458594 \mathrm{e}+06$ \\
7 & $2020-07-31$ & $1.431649 \mathrm{e}+06$ & $1.379320 \mathrm{e}+06$ & $1.483448 \mathrm{e}+06$ \\
8 & $2020-08-01$ & $1.458631 \mathrm{e}+06$ & $1.406738 \mathrm{e}+06$ & $1.510670 \mathrm{e}+06$ \\
9 & $2020-08-02$ & $1.485752 \mathrm{e}+06$ & $1.431273 \mathrm{e}+06$ & $1.535839 \mathrm{e}+06$ \\
10 & $2020-08-03$ & $1.512235 \mathrm{e}+06$ & $1.564263 \mathrm{e}+06$ \\
\hline
\end{tabular}

Fig. 7 Forecasting plot of coronavirus

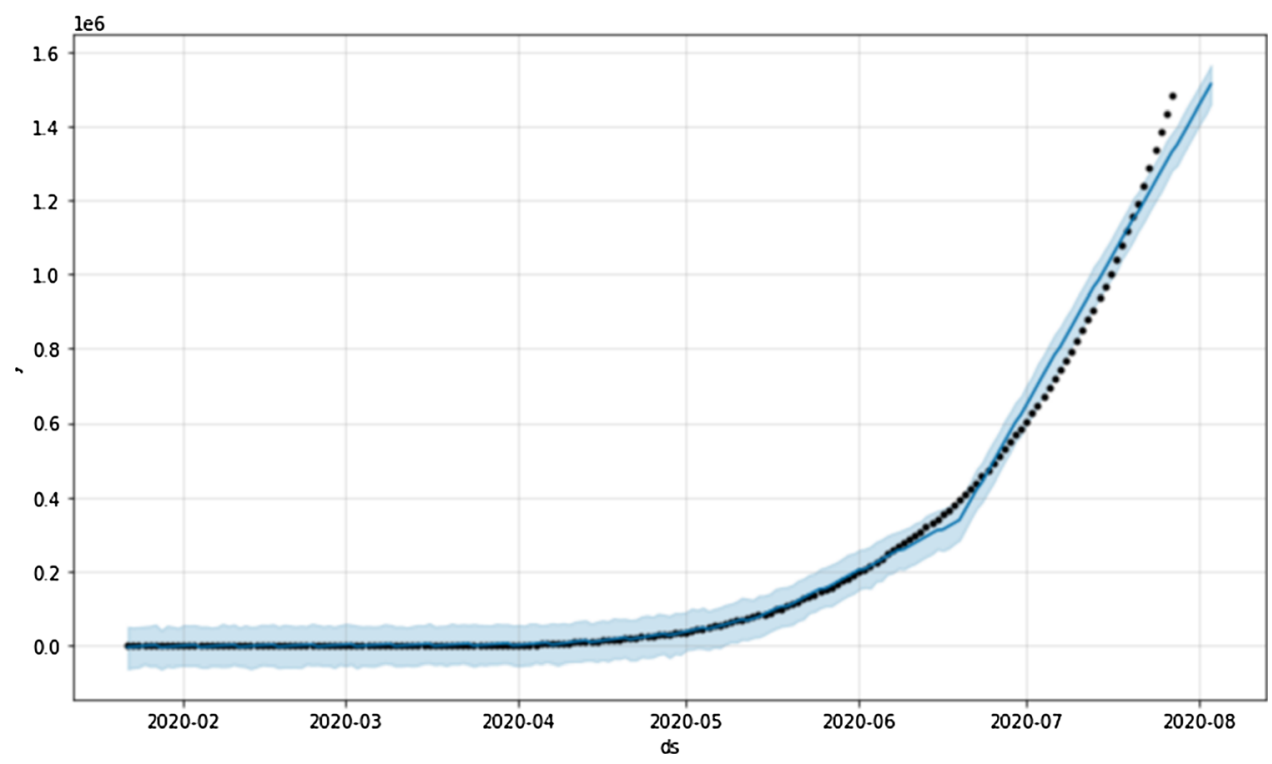


Fig. 8 Weekly forecast of coronavirus disease
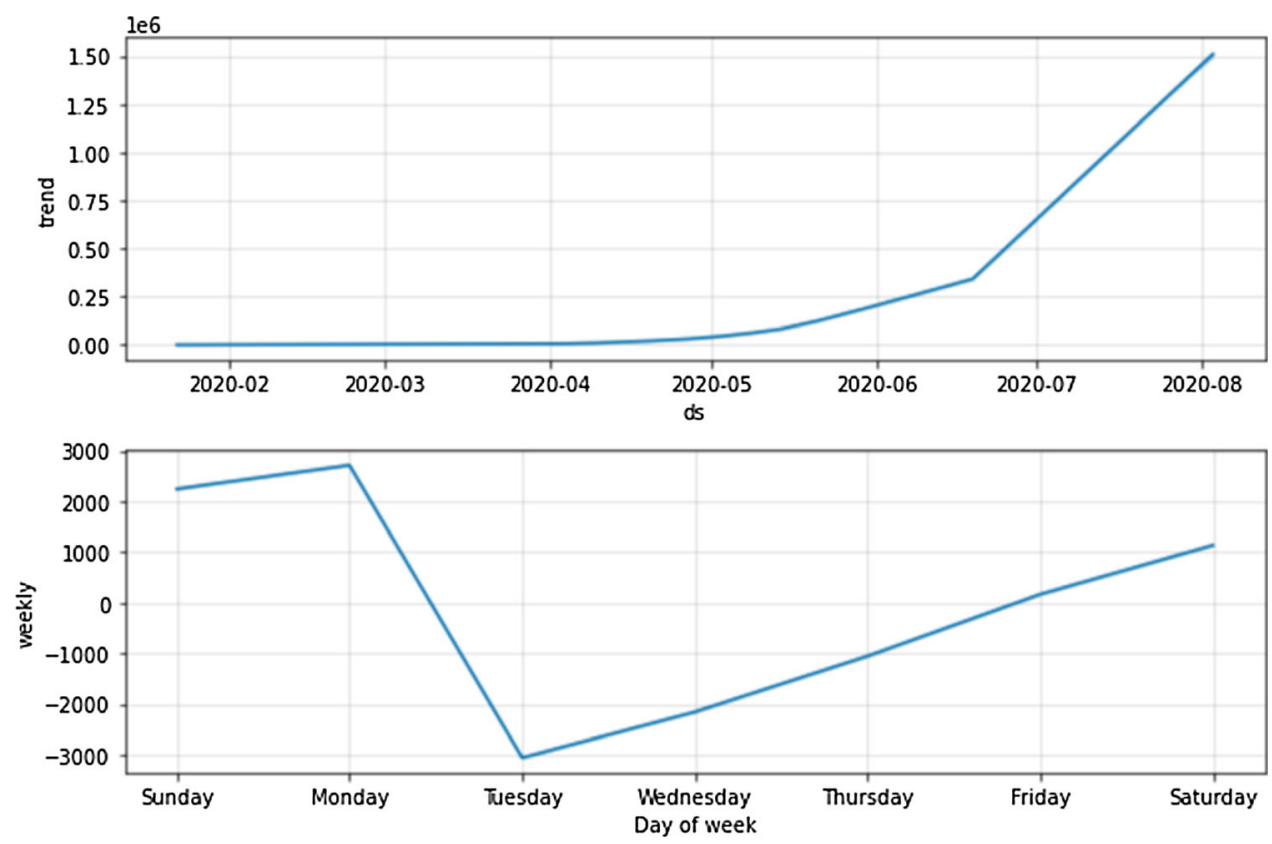

- $\quad c m(t)$ Total count of coronavirus cases at particular time period $\mathrm{t}$, that is equal to $c m(t)=H_{\mathrm{R}}(t)+H_{\mathrm{D}}(t)+R_{\mathrm{d}}(-$ $t)+D(t)$,

Total number of cases can also be calculated as:

$$
\begin{aligned}
c_{\mathrm{m}}(t) & =c_{\mathrm{m}}\left(t_{0}\right)+\int_{t_{0}}^{t} \frac{d\left(H_{\mathrm{R}}+H_{\mathrm{D}}+R_{\mathrm{d}}+D\right)}{\mathrm{d} t}(s) \mathrm{d} s \\
& =c_{\mathrm{m}}\left(t_{0}\right)+\int_{t_{0}}^{t} \theta(s) \gamma_{\mathrm{I}}(s) I(s) \mathrm{d} s
\end{aligned}
$$

- $\operatorname{dm}(\mathrm{t})$ : Total count of Coronavirus deaths at particular day t denoted by $D(t)$.

- $R_{0}$ and $R_{\mathrm{e}}$ : This represents fundamental propagation count and the effective multiplication count. $R_{0}$ (fundamental propagation count) is the average count of cases affected individual can spread to others in the contagious time. This count can increase according to populace. Besides, $R_{\mathrm{e}}(0)=R_{0}$. Normally, the spread of the malady eases back down if $R_{\mathrm{e}}(i, t)<1$. Figure 6 [11] shows the pictorial diagram of mathematical model of COVID-19.

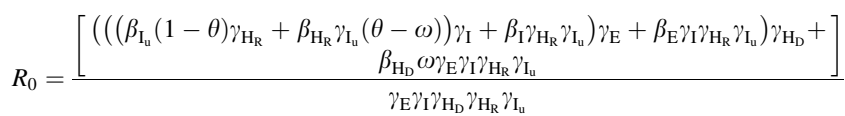

\section{Forecasting of Disease}

Coronavirus impacted economies and claimed a lot of lives. Forecasting of this disease can help to create awareness to arrange the maximum number of beds and ventilators to help the patients in future. As well as, if the number is higher, it will give a sense of responsibility to the citizens to help curb the spread of the disease.

\section{Algorithm}

1. Use the Kaggle coronavirus dataset of India.

2. Read the csv file and parse the dates.

3. Drop the unnecessary columns and replace the name of the columns.

4. Usually, the confirmed, recovered and deaths columns are provided in the dataset. Active cases can be easily computed by the formulae:

Active $=$ Confirmed $-($ Recovered + Deaths $)$

5. Use the library Prophet (from fbprophet import Prophet)

Prophet is a library in Python Created by Facebook for the time series analysis (forecasting). We need two columns (first-date(ds), second forecast_value $(y)$ ).

6. Build the model with a confidence level and fit the model for forecasting.

7. Make a future data frame and a desired value for the data to be forecasted.

8. The future forecasted gives an yhat actual value, also lower and upper values. Table 3 shows the lower and upper limit of prediction of virus cases. Figure 7 shows the forecast of the coronavirus.

9. Plot the future prediction and weekly forecast plot. We have forecasted for 20 extra days. Figure 8 shows the weekly forecast of the coronavirus. 


\section{Conclusion}

Previous work is explored and clarified in regard to their results from the scope of radiology to current innovations. These existing methods are not exact, time taking and are expensive. AI is a forthcoming and helpful apparatus to recognize early diseases and furthermore helps in checking the state of the contaminated patients. We have tried to analyze different AI techniques for detection of coronavirus. Mathematical models and forecasting can also help to predict the impact of this grave disease for helping the medical personnel to help the country at large. We present a novel approach by introducing new AI methods, mathematical modeling of coronavirus cases and forecasting the cases using python library.

\section{References}

1. Novel coronavirus-China-World Health Organization. https://www.who.int/csr/don/12-january-2020-novel-coronaviruschina/en/. Accessed in Mar 2020

2. C. Wang, P.W. Horby, F.G. Hayden, G.F. Gao, A novel coronavirus outbreak of global health concern. The Lancet 395, 470-473 (2020). https://doi.org/10.1016/S0140-6736(20)30185-9

3. Novel coronavirus (2019-ncov) World Health Organization. https://www.who.int/emergencies/diseases/novel-Coronavirus2019. Accessed in Mar 2020

4. L. Zhong, L. Mu, J. Li, J. Wang, Z. YinD, Liu, early prediction of the 2019 novel coronavirus outbreak in the mainland china based on simple mathematical model. IEEE Access 8, 51761-51769 (2020). https://doi.org/10.1109/ACCESS.2020.2979599
5. R. Vaishya, M. Javaid, I.H. Khan, A. Haleem, Artificial intelligence (AI) applications for COVID-19 pandemic. Diabetes Metab. Syndr. Clin. Res. Rev. 14(4), 337-339 (2020). https://doi.org/10.1016/j.dsx.2020.04.012

6. H. S. Maghdid, K. Z. Ghafoor, A. S. Sadiq, K. Curran, K. Rabie, A Novel AI-enabled Framework to Diagnose Coronavirus COVID-19 using Smartphone Embedded Sensors: Design Study. (2020). https://arxiv.org/abs/2003.07434

7. X. Li, D. Zhu, COVID-Xpert: An AI powered population screening of COVID-19 cases using chest radiography images. 2020. arXiv:2004.03042

8. M.I. Razzak, S. Naz, A. Zaib, Deep learning for medical image processing: overview, challenges and the future. Classif. BioApps Lect. Notes Comput. Vis. Biomech. 26, 323-350 (2017). https://doi.org/10.1007/978-3-319-65981-7_12

9. Y. Li, L. Xia, Coronavirus disease 2019 (COVID-19): role of chest CT in DIAGNOSIS AND MANagement. Am. J. Roentgenol. 214, 1280-1286 (2020). https://doi.org/10.22 14/AJR.20.22954

10. T. Ozturk, M. Talo, E.A. Yildirim, U.B. Baloglu, O. Yildirim, U.R. Acharya, Automated detection of COVID-19 cases using deep neural networks with X-ray images. Comput. Biol. Med. 121, 1-11 (2020). https://doi.org/10.1016/j.compbiomed.2020.10 3792

11. B. Ivorra, M.R. Ferrández, M. Vela-Pérez, A.M. Ramos, Mathematical modeling of the spread of the coronavirus disease 2019(COVID-19) taking into account the undetected infections. The case of China. Commun. Nonlinear Sci. Numer. Simulat. 88, 1-21 (2020). https://doi.org/10.1016/j.cnsns.2020.105303

12. B. Ivorra, D. Ngom, A.M. Ramos, Be-CoDiS: a mathematical model to predict the risk of human diseases spread between countries. Validation and application to the 2014 Ebola Virus Disease epidemic. Bull. Math. Biol. 77(9), 1668-1704 (2015). https://doi.org/10.1007/s11538-015-0100-x

Publisher's Note Springer Nature remains neutral with regard to jurisdictional claims in published maps and institutional affiliations. 